\title{
Note on CVL Plasma Field Equations
}

\author{
A. C. Hindmarsh UCRL-ID -105180 \\ DE91 002572
}

October 5, 1990

In [1], Boley describes the discretized equations for the magnetic field diffusion in the CVL plasma. In this note, these equations are derived in a different way (avoiding ghost points), and an error analysis is given.

Neglecting axial diffusion, the azimuthal field $B(r, z, t)$ is given by the following diffusion equation on $a_{1} \leq r \leq a_{2}, 0 \leq z \leq \ell, t \geq 0$ :

$$
\frac{\partial B}{\partial t}=\frac{\partial E}{\partial r}=\frac{\partial}{\partial r}\left[\frac{D}{r} \frac{\partial(r B)}{\partial r}\right] .
$$

The diffusion coefficient $D=D(r, z, B)$ is related to the conductivity $\sigma$ by $D=1 / \mu_{0} \sigma$. The boundary conditions are related to the plasma current $I_{p}$ and return current $I_{c}$ (which depend only on $t$ ) by

$$
B\left(a_{1}, z, t\right)=\frac{-\mu_{0}}{2 \pi a_{1}} I_{c}, \quad B\left(a_{2}, z, t\right)=\frac{\mu_{0}}{2 \pi a_{2}}\left(I_{p}-I_{c}\right) .
$$

These currents are in turn related to the laser voltage $V_{\ell}$ and the voltages $V_{a_{1}}, V_{a_{2}}$ at the radial edges by

$$
\left.\begin{array}{ll}
\dot{I}_{c} & =M_{10} V_{\ell}+M_{11} V_{a_{1}}-M_{12} V_{a_{2}} \\
\dot{I}_{p}-\dot{I}_{c} & =M_{20} V_{\ell}+M_{21} V_{a_{1}}-M_{22} V_{a_{2}}
\end{array}\right\}
$$

These voltages are related to $I_{p}$ and $E_{z}$ by

$$
V_{\ell}=V_{p k}^{r}+R_{p k}\left(I_{n s}-I_{p}\right)
$$

and

$$
V_{a_{i}}=\int_{0}^{\ell} E_{z}\left(a_{i}, z\right) d z+V_{e_{i}} \quad(i=1,2) .
$$


The discretization of (1) would be straightforward if it were not for the boundary conditions. Implicit in (2) is the requirement that at each radial edge $r=a_{i}$ the field is independent of $z$. Using a mesh of $N_{r}$ radial points $r_{i}$,

$$
a_{1}=r_{1}<r_{2}<\ldots<r_{N_{r}}=a_{2}
$$

and $N_{z}$ axial points $z_{j}$, let $B_{i j}$ be the discrete approximation to $B\left(r_{i}, z_{j}, t\right)$. For $i=1$ or $N_{r}$, $B_{i j}=B_{i}$ is independent of $j$. Denoting

$$
h_{i}=r_{i+1}-r_{i}, \quad r_{i+1 / 2}=\left(r_{i}+r_{i+1}\right) / 2
$$

and

$$
d(r, z)=D(r, z, B(r, z)) / r
$$

we discretize (1) at the radially interior points by setting

$$
E_{i+1 / 2, j}=d_{i+1 / 2, j}\left(\frac{r_{i+1} B_{i+1, j}-r_{i} B_{i j}}{h_{i}}\right)
$$

to approximate $E_{z}\left(r_{i+1 / 2}, z_{j}\right)$, and

$$
\dot{B}_{i j}=\frac{E_{i+1 / 2, j}-E_{i-1 / i, j}}{\left(h_{i}+h_{i-1}\right) / 2}
$$

for $i=2, \ldots, N_{r}-1$. Here we define

$$
\left.\begin{array}{r}
d_{i+1 / 2, j}=D\left(r_{i+1 / 2}, z_{j}, B_{i+1 / 2, j}\right) / r_{i+1 / 2} \\
B_{i+1 / 2, j} \equiv \frac{1}{2}\left(B_{i j}+B_{i+1, j}\right)
\end{array}\right\}
$$

to get $d=D / r$ at the radial midpoints. The equations (7)-(9) correspond to the standard secondorder central difference form for (1). (There are alternatives with the same order of accuracy, but no clear reason to prefer any one choice.)

The difficulty at the radial edges is in getting edge values for $E_{z}$,

$$
E_{z}\left(a_{i}, z\right)=\left.d\left(a_{i}, z\right) \frac{\partial(r B)}{\partial r}\right|_{a_{i}}(i=1,2) .
$$


A two-point one-sided difference for (10) is only first-order accurate, while a three-point one-sided difference would increase the bandwidth of the coupling in (8), which is tridiagonal. Instead, we look at a modification of a two-point difference. Consider the expression in (7) with $i=1$ :

$$
E_{3 / 2, j}=d_{3 / 2, j}\left(\frac{r_{2} B_{2, j}-r_{1} B_{1}}{h_{1}}\right) .
$$

Assuming $B_{1}$ and $B_{2, j}$ to be exact, this is a second-order accurate value for $E_{z}$ at $r_{3 / 2}$ :

$$
E_{3 / 2, j}=E_{z}\left(r_{3 / 2}, z_{j}\right)+O\left(h_{1}^{\prime}\right)
$$

For the exact values of $\mathrm{E}_{z}$, we also have

$$
E_{z}\left(r_{3 / 2}, z_{j}\right)=E_{z}\left(a_{1}, z_{j}\right)+\left.\frac{1}{2} h_{1} \frac{\partial E_{z}}{\partial r}\right|_{\left(a_{1}, z_{j}\right)}+O\left(h_{1}^{2}\right)
$$

Combining (11), (12), and (1) at $r=a_{1}$, we have

$$
E_{3 / 2, j}=E_{z}\left(a_{1}, z_{j}\right)+\frac{1}{2} h_{1} \dot{B}_{1}+O\left(h_{1}^{2}\right)
$$

This says that a value for $E_{z}\left(a_{1}, z_{j}\right)$, accurate to second order, is given by

$$
\tilde{E}_{1, j} \equiv E_{3 / 2, j}-\frac{1}{2} h_{1} \dot{B}_{1}
$$

In a similar way, a Taylor series for (7) with $i=N_{r}-1$ gives

$$
E_{N_{r}-1 / 2, j}=E_{z}\left(a_{2}, z_{j}\right)-\frac{1}{2} h_{N_{r}-1} \dot{B}_{N_{r}}+O\left(h_{N_{r}-1}^{2}\right)
$$

and thus leads to

$$
\tilde{E}_{N_{r, j}} \equiv E_{N_{r}-1 / 2, j}+\frac{1}{2} h_{N_{r}-1} \dot{B}_{N_{r}}
$$

as an accurate value for $E_{z}\left(a_{2}, z_{j}\right)$.

The appearance of the edge values of $\dot{B}$ in (14) and (15) means that the discrete representation of the boundary equations (2)-(5) will not be explicit at first, and will have to be solved for those 
values of $\dot{B}$. First, we form the discrete approximation to the edge voltages, with discrete sums in (5):

$$
V_{a_{1}}=\sum_{j} \ell_{j} \tilde{E}_{1, j}+V_{e_{1}}, \quad V_{a_{2}}=\sum_{j} \ell_{j} \tilde{E}_{N_{r, j}}+V_{e_{2}} .
$$

If we define explicit edge voltages

$$
\tilde{V}_{1}=\sum_{j} \ell_{j} E_{3 / 2, j}, \quad \tilde{V}_{2}=\sum_{j} \ell_{j} E_{N_{r}-1 / 2, j}
$$

then we have

$$
V_{a_{1}}=\tilde{V}_{1}-\frac{\ell}{2} h_{1} \dot{B}_{1}+V_{e_{1}}, \quad V_{a_{2}}=\tilde{V}_{2}+\frac{\ell}{2} h_{N_{r}-1} \dot{B}_{N_{r}}+V_{e_{2}}
$$

Next, differentiate (2) and combine with (3) to get

$$
\left[\begin{array}{c}
\frac{-2 \pi a_{1}}{\mu_{0}} \dot{B}_{1} \\
\frac{2 \pi a_{2}}{\mu_{0}} \dot{B}_{N_{r}}
\end{array}\right]=V_{\ell}\left[\begin{array}{l}
M_{10} \\
\operatorname{lv}_{20}
\end{array}\right]+\left[\begin{array}{ll}
M_{11} & M_{12} \\
M_{21} & M_{22}
\end{array}\right]\left[\begin{array}{c}
V_{a_{1}} \\
-V_{a_{2}}
\end{array}\right] .
$$

Inserting (17) into (18) and combining the $\dot{B}$ terms, we have

$$
\begin{array}{r}
{\left[\begin{array}{cc}
\frac{-2 \pi a_{1}}{\mu_{0}}+\frac{\ell}{2} h_{1} M_{11} & \frac{\ell}{2} h_{N_{r}-1} M_{12} \\
\frac{\ell}{2} h_{1} M_{21} & \frac{2 \pi a_{2}}{\mu_{0}}+\frac{\ell}{2} h_{N_{r}-1} M_{22}
\end{array}\right]\left[\begin{array}{c}
\dot{B}_{1} \\
\dot{B}_{N_{r}}
\end{array}\right]} \\
=V_{\ell}\left[\begin{array}{c}
M_{10} \\
M_{20}
\end{array}\right]+\left[\begin{array}{ll}
M_{11} & M_{12} \\
M_{21} & M_{22}
\end{array}\right]\left[\begin{array}{c}
\tilde{V}_{1}+V_{e_{1}} \\
-\tilde{V}_{2}-V_{e_{2}}
\end{array}\right] .
\end{array}
$$

Finally, eliminating $I_{p}$ from (2) and inserting into (4) gives

$$
I_{p}=\frac{2 \pi}{\mu_{0}}\left(a_{2} B_{N_{r}}-a_{1} B_{1}\right)
$$

and

$$
V_{\ell}=V_{p k}+R_{p k} I_{n s}-R_{p k} \frac{2 \pi}{\mu_{0}}\left(a_{2} B_{N_{r}}-a_{1} B_{1}\right)
$$

The $2 \times 2$ system (19), together with (16) and (7) for $\tilde{V}_{1}$ and $\tilde{V}_{2}$, and (20) for $V_{\ell}$, gives the time derivatives of the radial edge field values in terms of all the discrete field values. The equations (19) and the interior equations (8) comprise an ODE system in the $\left(N_{r}-2\right) \cdot N_{z}+2$ unknowns $\left\{B_{i j}, B_{i}, B_{N_{r}}\right\}$. 
The development of (19) suggests that the edge $\dot{B}$ values have second-order spatial accuracy, i.e., their errors go as the square of the mesh spacings. It is possible to prove this claim rigorously. Given a continuous field $B(r, z, t)$, and assuming that the discrete values $E_{i j}$ atc. are exact, we can define exact edge values of $E_{z}$ by $(10)$, form exact values $V_{a_{1}}^{e}, V_{a_{2}}^{e}$ of the edge voltages defined by (5), and ask how the resulting exact values $\dot{B}_{1}^{e}$ and $\dot{B}_{N_{r}}^{e}$, defined by (18), compare with the approximate values from (19). Let $\epsilon_{1}$ and $\epsilon_{2}$ be the errors in $\dot{B}_{1}$ and $\dot{B}_{N_{r}}$, respectively. Then on subtracting the exact version of (18) from (19), we have

$$
\left[\begin{array}{c}
\frac{-2 \pi a_{1}}{\mu_{0}} \epsilon_{1} \\
\frac{2 \pi a_{2}}{\mu_{0}} \epsilon_{2}
\end{array}\right]+\frac{\ell}{2}\left[\begin{array}{ll}
M_{11} & M_{12} \\
M_{21} & M_{22}
\end{array}\right]\left[\begin{array}{c}
h_{1} \dot{B}_{1} \\
h_{N_{r}-1} \dot{B}_{N_{r}}
\end{array}\right]=\left[\begin{array}{cc}
M_{11} & M_{12} \\
M_{21} & M_{22}
\end{array}\right]\left[\begin{array}{c}
\tilde{V}_{1}+V_{e_{1}}-V_{a_{1}}^{e} \\
-\tilde{V}_{2}-V_{e_{2}}+V_{a_{2}}^{e}
\end{array}\right]
$$

We have, from (5) and (16),

$$
\tilde{V}_{1}+V_{e_{1}}-V_{a_{1}}^{\mathrm{e}}=\sum_{j} \ell_{j} E_{3 / 2, j}-\int_{0}^{\ell} E_{z}\left(a_{1}, z\right) d z
$$

The Taylor series (1s) for $E_{3 / 2, j}$ (with exact values for $B$ and $\dot{B}$ ) gives

$$
\sum_{j} \ell_{j} E_{3 / 2, j}=\sum_{j} \ell_{j} E_{z}\left(a_{1}, z_{j}\right)+\frac{1}{2} \ell h_{1} \dot{B}_{1}^{e}+O\left(h_{1}^{2}\right) .
$$

Next, we assume that the quadrature rule

$$
\int_{0}^{\ell} f(z) d z \approx \sum_{j} \ell_{j} f\left(z_{j}\right)
$$

has at least second order, i.e., for smooth $f(z)$ it has error $=O\left((\Delta z)^{2}\right)$, where $\Delta z=\max \left(z_{j+1}-z_{j}\right)$. (For example, the Trapezoid Rule gives this accuracy.) Then we have

$$
\begin{aligned}
\tilde{V}_{1}+V_{e_{1}}-V_{a_{1}}^{e} & =\sum_{j} \ell_{j} E_{z}\left(a_{1} z_{j}\right)-\int_{0}^{\ell} E_{z}\left(a_{1}, z\right) d z+\frac{1}{2} \ell h_{1} \dot{B}_{1}^{e}+O\left(h_{1}^{2}\right) \\
& =\frac{1}{2} \ell h_{1} \dot{B}_{1}^{e}+C\left(h_{1}^{2}\right)+O\left((\Delta z)^{2}\right) .
\end{aligned}
$$

Similarly, the analogous Taylor series for $E_{N_{r}-1 / 2, j}$ gives

$$
-\tilde{V}_{2}-V_{e_{2}}+V_{a_{2}}^{e}=\frac{1}{2} \ell h_{N_{r}-1} \dot{B}_{N_{r}}^{e}+O\left(h_{N_{r}-1}^{2}\right)+O\left((\Delta z)^{2}\right) .
$$


Substituting into (21), and defining $\Delta r=\max \left(h_{i}\right)$, we obtain

$$
\begin{aligned}
{\left[\begin{array}{c}
\frac{-2 \pi a_{1}}{\mu_{0}} \epsilon_{1} \\
\frac{2 \pi a_{2}}{\mu_{0}} \epsilon_{2}
\end{array}\right] } & =\left[\begin{array}{ll}
M_{11} & M_{12} \\
M_{21} & M_{22}
\end{array}\right]\left[\begin{array}{l}
\frac{1}{2} \ell h_{1} \dot{B}_{1}^{e}-\frac{1}{2} \ell h_{1} \dot{B}_{1} \\
\frac{1}{2} \ell h_{N_{r}-1} \dot{B}_{N_{r}}^{e}-\frac{1}{2} \ell h_{N_{r}-1} \dot{B}_{N_{r}}
\end{array}\right]+O\left((\Delta r)^{2}\right)+O\left((\Delta z)^{2}\right) \\
& =-\frac{1}{2} \ell\left[\begin{array}{ll}
M_{11} & M_{12} \\
M_{21} & M_{22^{\prime}}
\end{array}\right]\left[\begin{array}{l}
h_{1} \epsilon_{1} \\
h_{N_{r}-1} \epsilon_{2}
\end{array}\right]+O\left((\Delta r)^{2}+(\Delta z)^{2}\right),
\end{aligned}
$$

or

$$
\left[\begin{array}{cc}
\frac{-2 \pi a_{1}}{\mu_{0}}+\frac{\ell}{2} h_{1} M_{11} & \frac{\ell}{2} h_{N_{r}-1} M_{12} \\
\frac{\ell}{2} h_{1} M_{21} & \frac{2 \pi a_{2}}{\mu_{0}}+\frac{\ell}{2} h_{N_{r-1}} M_{22}
\end{array}\right]\left[\begin{array}{c}
\epsilon_{1} \\
\epsilon_{2}
\end{array}\right]=O\left((\Delta r)^{2}+(\Delta z)^{2}\right) .
$$

On applying the inverse of this $2 \times 2$ matrix (which is clearly nonsingular for all sufficiently small values of $h_{1}$ and $\left.h_{N_{r}-1}\right)$, we conclude that $\epsilon_{1}$ and $\epsilon_{2}$ are $O\left((\Delta r)^{2}+(\Delta z)^{2}\right)$. The central difference expressions in (8) are also accurate to $O\left((\Delta r)^{2}\right)$. Thus we can ronclude that all of the discrete $\dot{B}$ values from (8) and (19) are second-order accurate, i.e., have errors $=O\left((\Delta r)^{2}+(\Delta z)^{2}\right)$.

\section{Reference}

[1] C. D. Boley, "New Capabilities in the Circe Model of the Copper Laser Modulator and Plasma Load," Memorandum CLT 90-12, February 15, 1990. 

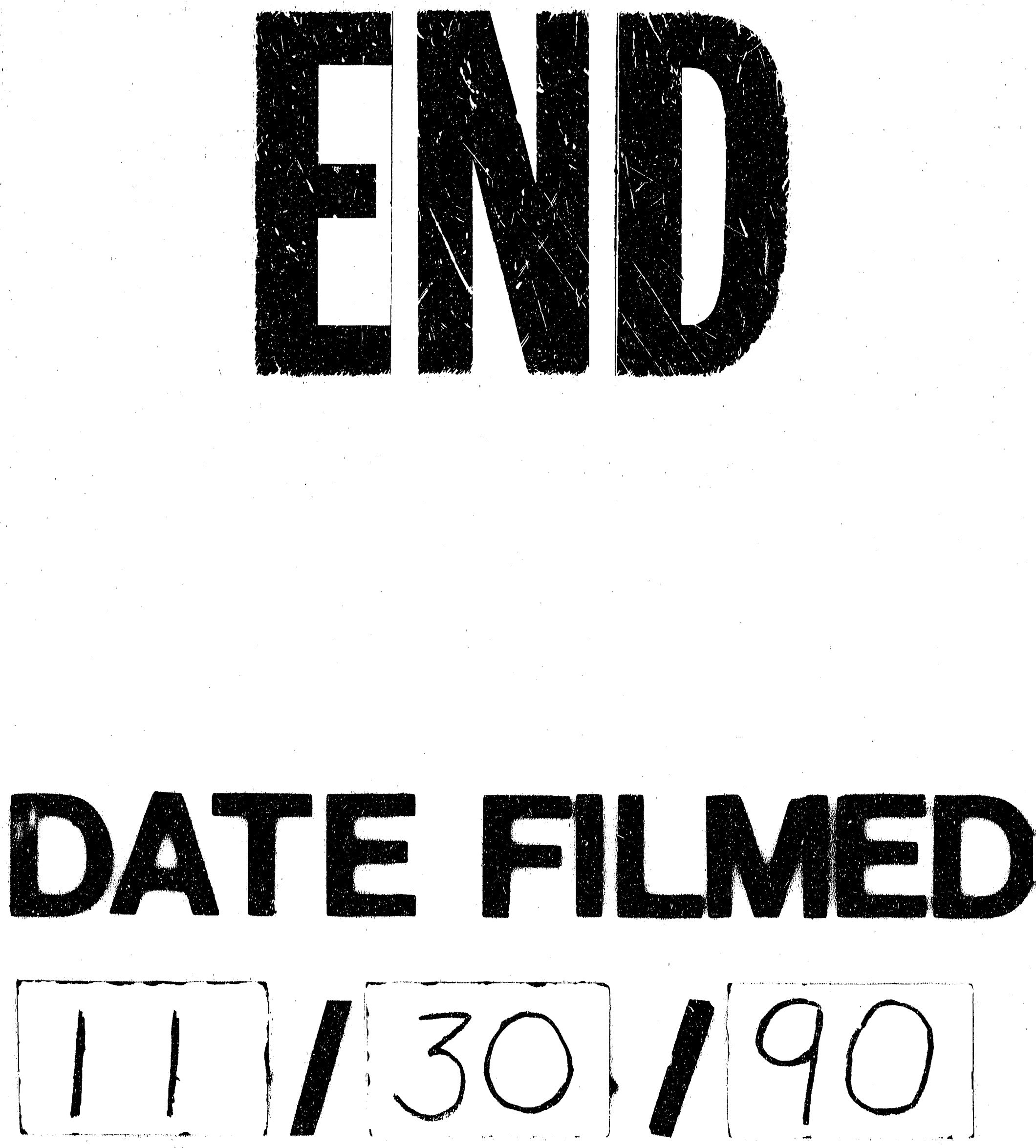
